\title{
OPEN Structure of Nora virus at $2.7 \AA$ resolution and implications for receptor binding, capsid stability and taxonomy
}

\author{
Pasi Laurinmäki $\mathbb{D}^{1,2,7}$, Shabih Shakeel $\mathbb{D}^{1,2,5,7}$, Jens-Ola Ekström ${ }^{3,4,7}$, \\ Pezhman Mohammadi (iD) ${ }^{1,6}$, Dan Hultmark (iD ${ }^{3,4}$ \& Sarah J. Butcher ${ }^{1,2}{ }^{\square}$
}

Nora virus, a virus of Drosophila, encapsidates one of the largest single-stranded RNA virus genomes known. Its taxonomic affinity is uncertain as it has a picornavirus-like cassette of enzymes for virus replication, but the capsid structure was at the time for genome publication unknown. By solving the structure of the virus, and through sequence comparison, we clear up this taxonomic ambiguity in the invertebrate RNA virosphere. Despite the lack of detectable similarity in the amino acid sequences, the $2.7 \AA ̊$ resolution cryoEM map showed Nora virus to have $\mathrm{T}=1$ symmetry with the characteristic capsid protein $\beta$-barrels found in all the viruses in the Picornavirales order. Strikingly, $\alpha$-helical bundles formed from the extended C-termini of capsid protein VP4B and VP4C protrude from the capsid surface. They are similar to signalling molecule folds and implicated in virus entry. Unlike other viruses of Picornavirales, no intra-pentamer stabilizing annulus was seen, instead the intra-pentamer stability comes from the interaction of VP4C and VP4B N-termini. Finally, intertwining of the N-termini of two-fold symmetry-related VP4A capsid proteins and RNA, provides inter-pentamer stability. Based on its distinct structural elements and the genetic distance to other picorna-like viruses we propose that Nora virus, and a small group of related viruses, should have its own family within the order Picornavirales.

Nora virus is a positive-sense, single-stranded RNA virus infecting different Drosophila species including Drosophila melanogaster and Drosophila simulans ${ }^{1}$. The virus infects the midgut cells and the infection is transmitted vertically by the fecal-oral route. Interestingly, the infected insects exhibit no obvious pathological effects ${ }^{2-5}$. Nora virus has several characteristic features of the order Picornavirales ${ }^{6}$, such as an isometric capsid about $30 \mathrm{~nm}$ in diameter and a replicative cassette that includes an RNA helicase, a protease and an RNA-dependent RNA polymerase ${ }^{1}$. Based on the conserved polymerase and helicase sequences, Nora virus is similar to viruses in the Picornavirales families Iflaviridae, Secoviridae, Picornaviridae and Dicistroviridae ${ }^{1,7,8}$. However, uniquely, the Nora virus genome is organised into four open reading frames (ORF), and for a Picornavirales virus it has a relatively large RNA genome of 12,333 nucleotides ${ }^{1,9}$ (Fig. S1). It shares these characteristics with a small group of Nora-like viruses, most of them isolated from different insects ${ }^{1,7,8,10,11}$.

Nora virus ORF4, situated at the 3'-terminal end of the genome, encodes a 931 amino acid-long polyprotein. The translated polyprotein is cleaved at two positions (264 aa and $515 \mathrm{aa}$ ) post-translationally by a virus-encoded protease to form the virion's mature structural proteins VP4A $(29 \mathrm{kDa})$, VP4B $(28 \mathrm{kDa})$ and VP4C $(48 \mathrm{kDa})^{5,9}$. The proteins encoded by ORF4 have no significant sequence similarity to the capsid proteins of other picorna-like viruses or to each other. However, structure predictions indicated a possible picorna-like jelly roll fold for VP4A and VP4B. In contrast, VP4C was predicted to have a mainly a-helical secondary structure ${ }^{1}$. Furthermore, VP4C is susceptible to proteolysis and is fragmented in virions isolated from fly feces, which begs the question whether

${ }^{1}$ HiLIFE-Institute of Biotechnology, University of Helsinki, Viikinkaari 9, P.O. Box 56, 00014 Helsinki, Finland. ${ }^{2}$ Molecular and Integrative Biosciences Research Programme, Faculty of Biological and Environmental Sciences, University of Helsinki, Viikinkaari 9, P.O. Box 56, 00014 Helsinki, Finland. ${ }^{3}$ Department of Molecular Biology, Umeå University, 90187 Umeå, Sweden. ${ }^{~ I n s t i t u t e ~ o f ~ B i o s c i e n c e s ~ a n d ~ M e d i c a l ~ T e c h n o l o g y, ~ B i o M e d i T e c h, ~}$ University of Tampere, 33014 Tampere, Finland. ${ }^{5}$ Present address: MRC Laboratory of Molecular Biology, Francis Crick Avenue, Cambridge CB2 00H, UK. ${ }^{6}$ Present address: VTT Technical Research Centre of Finland Ltd., 02044 Espoo, Finland. ${ }^{7}$ These authors contributed equally: Pasi Laurinmäki, Shabih Shakeel and Jens-Ola Ekström. ${ }^{\boxplus}$ email: sarah.butcher@helsinki.fi 
it has a structural role or not $^{9}$. Additionally, trace amounts of the VP3 protein from ORF3 have been identified in purified Nora virus particles, isolated from feces. VP3 is not required for virus assembly, but stabilizes the capsid against heat and protease treatment. It is unclear whether or not it is physically integrated into the capsid ${ }^{9,12}$. We present the Nora virus capsid structure at $2.7 \AA$ resolution, using electron cryo-microscopy (cryoEM) and icosahedral image reconstruction, which helps us to understand the architecture of this virus, to investigate the presence of VP3 in the capsid and to investigate the function of VP4C.

\section{Results and discussion}

We determined a $2.7 \AA$ resolution Nora virus structure using electron cryo-microscopy and single particle image analysis (Fig. 1). The models of capsid proteins VP4A, VP4B and VP4C were built de novo to generate an atomic model of Nora virus constrained by the density from the reconstruction (Fig. 2 and Table 1). The Nora virus reconstruction displays an icosahedrally- symmetric particle with a $T=1$ (pseudo $T=3$ ) triangulation number; a capsid architecture previously described as one of the main characteristics of the order Picornavirales (Fig. 1 $\mathrm{c}$ and Fig. 2a) ${ }^{6}$. The Nora virus capsid asymmetric unit is built from three subunits, a single copy each of VP4A, VP4B and VP4C (Fig. 2c). Major domains of all the three subunits are $\beta$-sheet jelly rolls (Fig. 2a,c) ${ }^{6}$. The five-fold vertices are composed of VP4C, the three-fold facets are composed of VP4A and VP4B and the two-fold edges are composed of VP4A (Fig. 2a,b). We were unambiguously able to trace the C- $\alpha$ backbone for VP4A (residues 1-249 out of 264), for VP4B (2-242 out of 251) and for VP4C (1-364 out of 416). The disordered C-termini regions are presumably exposed on the capsid surface, as the last visible residues contribute to the striking protrusions that circle the pentamers composed of both the VP4B and the VP4C C-termini. Hence, the termini are potentially susceptible to host protease attack, explaining the observed shortening of VP4C in virus isolates from fly feces ${ }^{9}$. No additional density for VP3 was identified in the capsid density. As only trace amounts of VP3 have been detected previously in isolated virus, if associated with the capsid, it is probably in non-stoichiometric amounts and may also be disordered, resulting in it being averaged out during icosahedral averaging.

The evolutionary expansion of the Nora virus capsid. The Nora virus capsid size was compared with the capsids from representative viruses within the order Picornavirales: Dicistroviridae, Iflaviridae, Secoviridae and Picornaviridae. The inner shell of the Nora virus capsid had the highest diameter of all of them (Table 2, Fig. 3), in line with the need to accommodate the unusually large $12 \mathrm{~kb}$ genome. The volume available inside the capsid per nucleotide of the genome varied from 0.52 to 0.67 (Table 2). Interestingly, the buried surface area of the Nora virus capsid is smaller than all the others, suggesting a relative expansion of the capsid during evolution accomplished through conformational change (Table 2, Fig. 3). Additionally, the VP4C $\beta$-barrels around the vertices lay flatter on the capsid surface compared to the more tangential arrangement of $\beta$-barrels in the other viruses (Fig. 3). This translation of the $\beta$-barrels appears to be the most obvious cause of capsid expansion in Nora virus. In conclusion, the capsid size comparison shows that while there is significant variation in the capsid sizes of Picornavirales, the Nora virus capsid is the largest thus far. The expansion allows the encapsidation of the large Nora virus genome. There are potentially many other factors that could also affect the packaging density, as the percentage difference in the ratio of genome length to capsid volume is not linear. One such possibility is that the secondary and tertiary structure of the RNA varies. Another possibility is that the presence of counterions may affect the density. A third possibility is that the number of amino acid residues actually included in the atomic models may not account for the full-length protein present in the capsids, thus the volume allocated to the genome maybe overestimated. In the case of Nora virus only one missing amino acid residue in VP4B is thought to be internal, the other missing amino acids in all three proteins are thought to be external. Hence, the volume occupied by 60 amino acids in total would reduce the volume available to the Nora virus genome. If trace amounts of VP3 are present within the capsid, these too would reduce the volume available to the genome.

Potential receptor binding region. Structural alignment with DALI (13) showed that VP4A is most similar to FMDV VP2 (Z-score: 12.8; rmsd: 3.0; PDB: 1fmd; family: Picornaviridae; genus: Aphthovirus), VP4B is most similar to human Aichi virus VP0 (Z-score: 10.6; rmsd: 3.2; PDB: 5gka; family: Picornaviridae; genus: Kobuvirus) and VP4C is most similar to hepatitis A virus VP3 (Z-score: 11.0; rmsd: 3.2; PDB: 4qpg; family: Picornaviridae; genus: Hepatovirus). The $\beta$-barrels account for the alignment. However, the most prominent capsid surface features are the 60 mainly $\alpha$-helical protrusions that are situated at the interface between VP4C contributing most of the residues and VP4B contributing one $\alpha$-helix (Fig. 2b-d). These surface protrusions are significantly different from that of other insect viruses: triatoma virus, Israel acute paralysis virus and slow bee paralysis virus where the surface protrusions mostly consist of $\beta$ strands (Fig. 4). We entered the structured VP4C residues 287-364 into a DALI alignment. Strikingly, it had structural similarity to inositol 1, 4, 5-triphosphate receptor type 1 (PDB: $3 \mathrm{UJO})$, $\alpha-N$-acetylglucosaminidase (PDB: $4 \mathrm{XWH}$ ) and the non-structural ORF 12 of the virulent lactococcal phage p2 (PDB: 3D8L). All these three molecules are involved in signalling, suggesting that the Nora virus surface protrusion may have a similar role, binding to a cell surface receptor and causing downstream signalling to bolster virus entry. Additionally, the surface charge distribution of these protrusions shows a positive patch which, similar to the C-terminal extension of S protein in cowpea mosaic virus, may have a role in capsid assembly by stabilizing the formation of pentamers during assembly ${ }^{14}$.

Stabilization of the capsid. A very distinct feature in Nora virus is the lack of the annulus found below the vertices in dicistrovirus, picornavirus and iflavirus capsids formed by interaction of $5 \mathrm{~N}$-termini from the VP3 capsid proteins. Annulus formation is a primary requirement for intra-pentamer stability in those capsids. It is fulfilled in Nora virus by extensive interaction of VP4B and VP4C N-termini around the five-fold but at a much greater distance from the five-fold axis of symmetry (Fig. 5). 

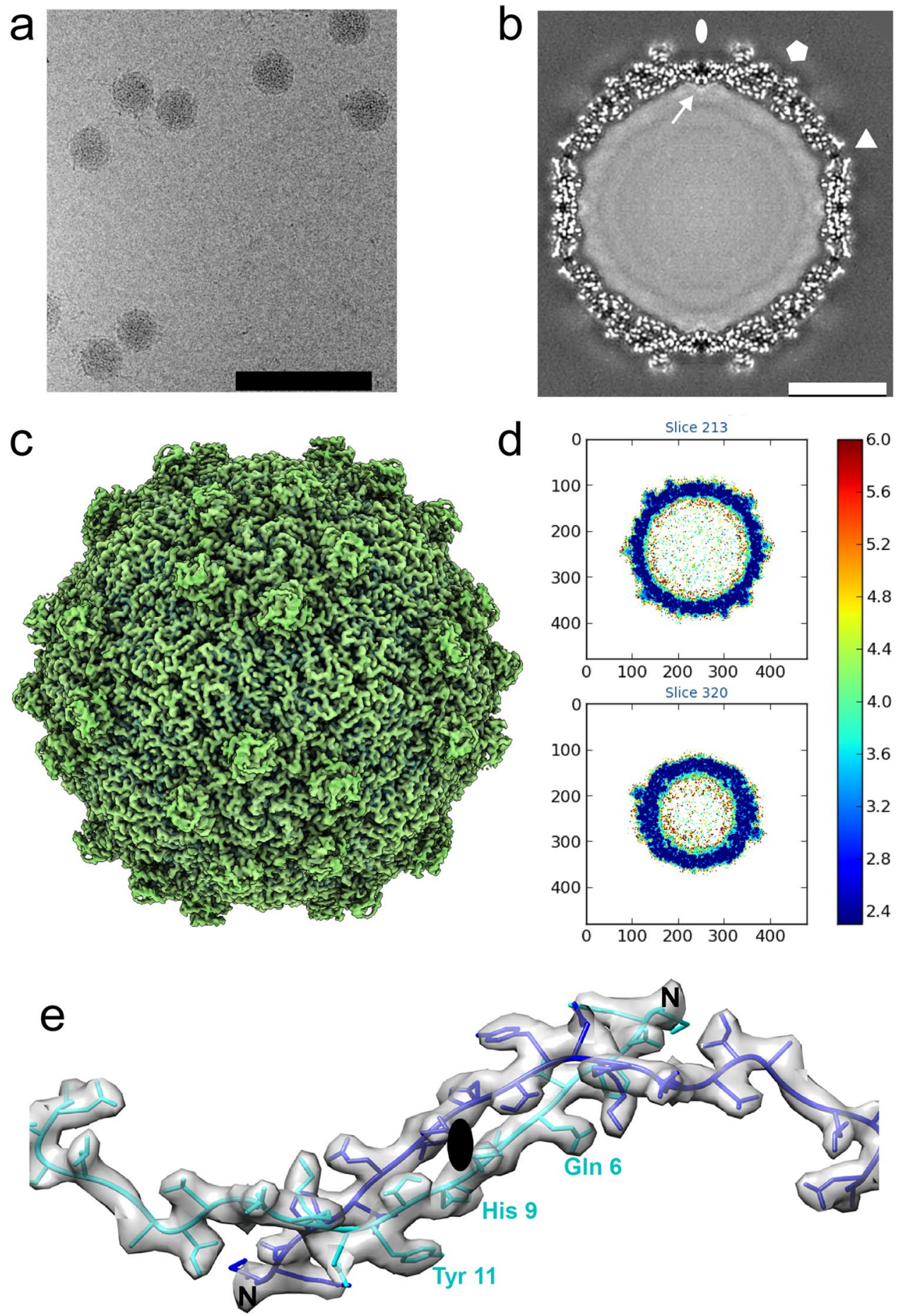

Figure 1. Nora virus cryoEM reconstruction. (a) Representative cryoEM micrograph of Nora virus. Scale bar is $100 \mathrm{~nm}$. (b) Central section through the Nora virus reconstruction with protein white. White ellipse, pentagon and triangle indicate positions of symmetry axes. White arrow: strongest ordered RNA density under the twofold symmetry axis. Scale bar is $10 \mathrm{~nm}$. (c) Isosurface representation of Nora virus density rendered at $3 \mathrm{SD}$ above the mean. (d) Local resolution of the EM density map calculated using ResMap ${ }^{36}, 2$ slices each of $1.06 \AA$ thick are shown. The upper slice is $28.62 \AA$ from the center of the particle, the lower slice is $84.8 \AA$ from the center of the particle (which would be section 240) Color key units are in $\AA$. (e) Representative atomic model fit of VP4A in the vicinity of the two-fold symmetry axis. Grey semi-transparent surface: reconstructed volume rendered at 3 standard deviations above the mean. Cyan and blue ribbons: atomic models of $25 \mathrm{~N}$-terminal residues of two symmetry related VP4As. N-termini marked with a letter N, three AA residues are annotated for one VP4A. 
a

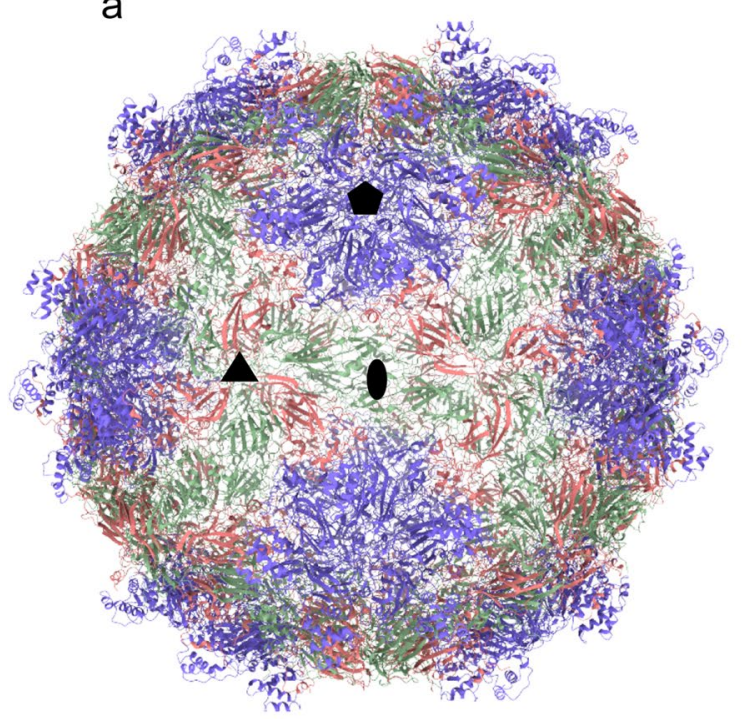

b

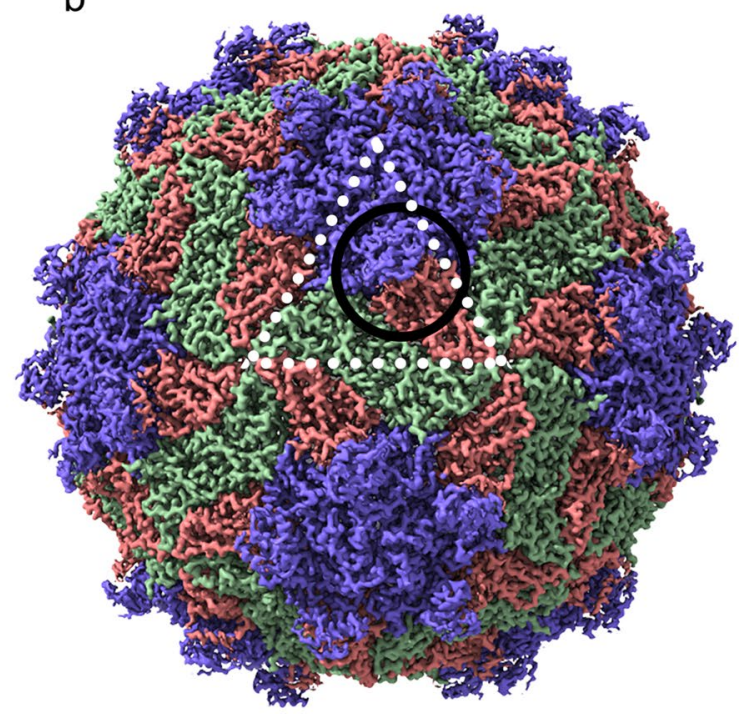

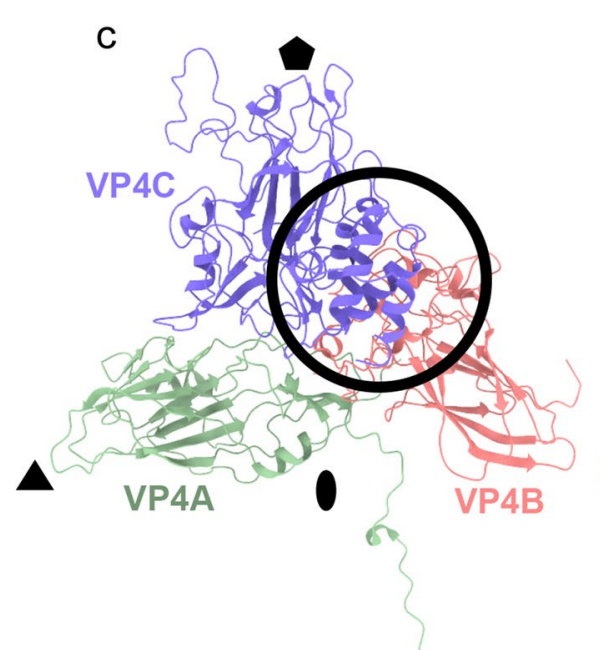

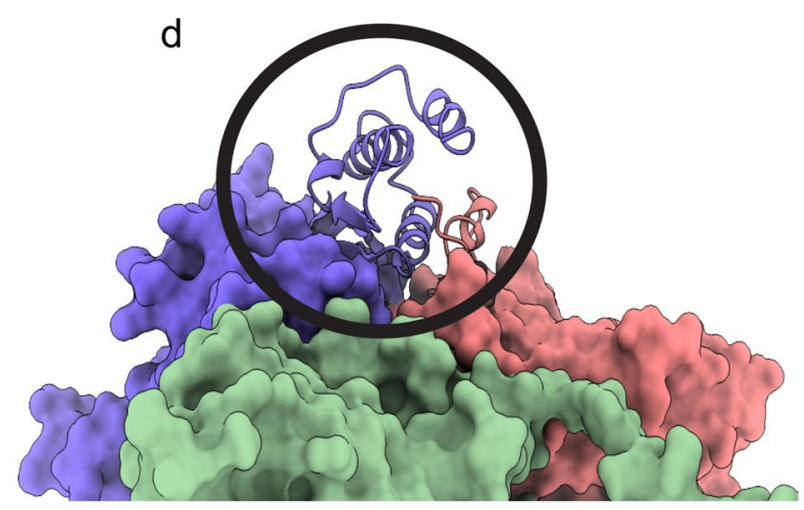

Figure 2. Architecture of Nora virus capsid. (a) Atomic model of Nora virus capsid in ribbon. (b) Isosurface representation of Nora virus density rendered at $3 \mathrm{SD}$ above the mean. The map was zoned and coloured according to model with distance of $5 \AA$ using chimeraX. (c) Capsid asymmetric unit in ribbon. (d) Close-up of surface protrusion shown as ribbon. Green: VP4A; pink: VP4B; and blue: VP4C. Black ellipse, pentagon and triangles indicate positions of two-fold, five-fold and three-fold symmetry axes in (a) and (c). The surface protrusion is encircled in $(\mathbf{b})-(\mathbf{d})$.

The Nora virus capsid seems to utilize the N-termini of VP4A to provide interpentamer stability by spanning from the three-fold axis to the two-fold axis, a feature lacking in most other viruses of Picornavirales except human parechoviruses $(\mathrm{HPeVs})^{13,14}$. However, unlike $\mathrm{HPeVs}$, there is a crossover of the VP4A N-terminus (residues 1-13) with a symmetry-equivalent $\mathrm{N}$-terminus of another VP4A from the neighbouring pentamer at the two-fold axis (Fig. 1e). Additionally, two molecules of VP4A sit either side of the two-fold axis of symmetry. At this position, there are two a-helices (aa 109-117), one contributed by each molecule on the capsid surface (Fig. 2c). Although this $\alpha$-helical feature is common in other Picornavirales, in Nora virus, they are further apart (Fig. 6a), for instance, the middle residue in the helix, T114 CB is $6.9 \AA$ from its symmetry related atom in comparison to $4.6 \AA$ for Q94 CB of VP2 in coxsackievirus A 9 (CVA9). These helices are important for capsid stability and separate during RNA egress in Picornaviridae ${ }^{15-18}$. In Nora virus, the interface between the VP4A molecules is strengthened by the tight interaction of the VP4A N-termini in the inner surface (Figs. 1e, 6a). Such deviation from the common theme raises the question of how the genome release occurs in Nora virus, and this will require further investigation.

Capsid protein-RNA interactions. The symmetry-related, positively-charged histidines (residue number 9) of the two VP4A N-termini on the two-fold axis closely interact with the ssRNA genome. This causes a 


\begin{tabular}{|c|c|c|c|c|}
\hline & Noravirus & & & \\
\hline \multicolumn{5}{|l|}{ Data collection and processing } \\
\hline Voltage (keV) & 300 & & & \\
\hline Electron exposure $\left(\mathrm{e}^{-} / \AA^{2}\right)$ & 40 & & & \\
\hline Defocus range $(\mu \mathrm{m})$ & $0.5-1.5$ & & & \\
\hline Pixel size & 1.06 & & & \\
\hline Symmetry imposed & I & & & \\
\hline Number of movies collected & 3516 & & & \\
\hline Final particle images (no.) & 16,131 & & & \\
\hline Map resolution $(\AA)$ & 2.7 & & & \\
\hline \multirow[t]{2}{*}{ Map resolution range $(\AA)$} & $2.4-6$ & & & \\
\hline & VP4A & VP4B & VP4C & VP4ABC \\
\hline \multicolumn{5}{|l|}{ Refinement } \\
\hline Map cross correlation (around atoms) & 0.855 & 0.813 & 0.789 & 0.805 \\
\hline rmsd (bonds) & 0.01 & 0.01 & 0.01 & 0.01 \\
\hline rmsd (angles) & 1.08 & 1.33 & 1.18 & 1.27 \\
\hline All-atom clashscore & 2.87 & 3.16 & 3.24 & 3.49 \\
\hline Ramachandran outliers & $0.81 \%$ & $0.84 \%$ & $1.10 \%$ & $0.83 \%$ \\
\hline Ramachandran allowed & $7.29 \%$ & $13.81 \%$ & $11.88 \%$ & $12.15 \%$ \\
\hline Ramachandran favoured & $91.90 \%$ & $85.36 \%$ & $87.02 \%$ & $87.03 \%$ \\
\hline
\end{tabular}

Table 1. CryoEM data collection and model refinement statistics.

\begin{tabular}{|c|c|c|c|c|c|c|c|c|c|}
\hline Family $^{a}$ & Genus & Virus name & PDB id & $\begin{array}{l}\text { Inner diameter } \\
(\mathrm{nm})\end{array}$ & $\begin{array}{l}\text { Shell thickness } \\
(\mathrm{nm})\end{array}$ & $\begin{array}{l}\text { Inner volume } \\
\left(10^{3} \mathrm{~nm}\right)\end{array}$ & $\begin{array}{l}\text { Genome size } \\
\text { (kb) }\end{array}$ & $\begin{array}{l}\text { Volume per } \\
\text { nucleotide } \\
\left(\mathrm{nm}^{3}\right)\end{array}$ & $\begin{array}{l}\text { Buried surface } \\
\text { area }\left(10^{3} \mathrm{~nm}\right)\end{array}$ \\
\hline Unclassified & Unclassified & Nora virus & $5 \mathrm{~mm} 2$ & 24.2 & 10.1 & 7.4 & 12.5 & 0.59 & 2.0 \\
\hline Dicistroviridae & Triatovirus & Triatoma virus & 3nap & 22.8 & 10.8 & 6.2 & 9.2 & 0.67 & 2.5 \\
\hline Dicistroviridae & Aparavirus & $\begin{array}{l}\text { Israel acute } \\
\text { paralysis virus }\end{array}$ & $5 \mathrm{cdc}$ & 22.8 & 11.6 & 6.2 & 9.7 & 0.64 & 2.3 \\
\hline Dicistroviridae & Cripavirus & $\begin{array}{l}\text { Cricket paralysis } \\
\text { virus }\end{array}$ & $1 \mathrm{~b} 35$ & 21.6 & 12.8 & 5.3 & 9.4 & 0.56 & 3.0 \\
\hline Iflaviridae & Iflavirus & $\begin{array}{l}\text { Slow bee paralysis } \\
\text { virus }\end{array}$ & $5 j 98$ & 22.8 & 14.6 & 6.2 & 9.5 & 0.65 & 2.1 \\
\hline Picornaviridae & Enterovirus & $\begin{array}{l}\text { Coxsackievirus } \\
\text { A9 }\end{array}$ & $1 \mathrm{~d} 4 \mathrm{~m}$ & 19.6 & 13.6 & 3.9 & 7.6 & 0.52 & 2.6 \\
\hline
\end{tabular}

Table 2. Comparison of capsid size inner volumes, genome sizes and buried surface area among

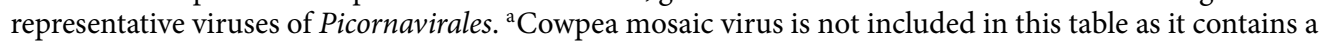
bipartite genome with each genome packed in a separate capsid. ${ }^{\text {b}} 200$ nucleotides are added to each genome for the poly-A tail.

localised condensation of RNA density in this region around the twofold symmetry axis (Figs. 1b, 6). Arginine (residue number 78) and lysine (residue number 224) add additional positive charge in close proximity, thereby further stabilizing the electrostatic interactions between the capsid protein and the RNA (Fig. 6). This location of RNA condensation is in contrast to the one found in HPeVs where it occurs around the vertices ${ }^{19,20}$. RNA-protein contacts around the twofold symmetry axes have been reported in several Picornavirales including cowpea mosaic virus containing RNA-2, in rhinovirus A2 and in CVA9. However, not with this particular distribution ${ }^{16,21,22}$. Hence, both the viral assembly and RNA release process may occur by different means compared to other Picornavirales. In the future, the RNA structure could possibly be studied further by asymmetric reconstruction of the capsids to high resolution, but the current data set was too small for this endeavour, probably requiring at least tenfold more particles as was done for bacteriophage MS2 $2^{23}$.

Phylogenetic analysis. Sequences related to the Nora virus capsid proteins can be identified in a number of virus-like sequences in the databases (Fig. 7 and Fig. S1). Based on the conserved RNA-dependent RNA polymerase sequences, Shi et al. ${ }^{8}$ have previously defined a "Nora Virus Related Clade" of viruses, but we found that Nora-like capsid protein sequences are only present in a subset of these viruses. This Nora-like subset forms a well-defined monophyletic group with a conserved genome organization (Fig. 7 and Fig. S1a), which is different from other members of the clade (Fig. S1b). The Nora-like viruses have generally been isolated from insects, with the exception of one isolate from a spider (T. maxillosa) and one from a sea anemone (A. equina). The capsid 


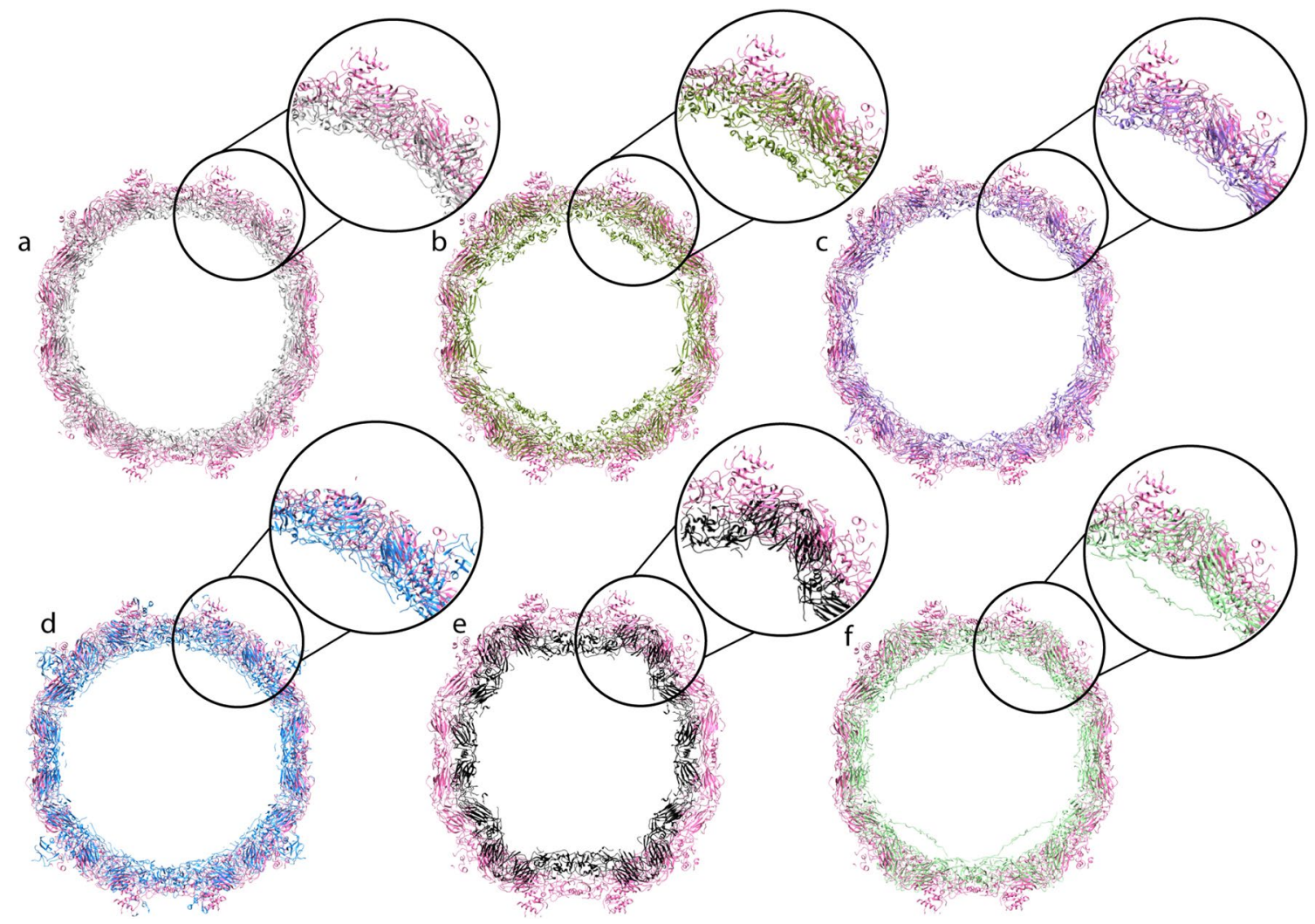

Figure 3. Capsid size comparison. Central cross-sections of Nora virus capsid (pink) versus (a) Triatoma (PDB: 3nap; Dicistroviridae; grey), (b) cricket paralysis virus (PDB: 1b35; Dicistroviridae, dark green), (c) Israel acute paralysis virus (PDB: 5cdc; Dicistroviridae, purple), (d) slow bee paralysis virus (PDB: 5j98; Iflaviridae, blue), (e) cowpea mosaic virus (PDB: 1ny7; Secoviridae, black), (f) coxsackievirus A9 (PDB: 1d4m; Picornaviridae, green). Nora virus has a larger diameter, thinner, capsid shell than the other viruses which is most pronounced around the two-fold axis, e.g. on the equator in this view. The $\beta$-barrels of VP4C around the vertices lie flatter on the capsid surface compared to equivalent barrels of picornaviruses. Overall, the capsid comparison shows that the Nora virus has undergone drastic expansion by means of translation as well as rotation of capsid proteins.

protein sequences are well conserved among the members of this group, suggesting that the structure described here is well adapted to the genome size and/or the biology of these viruses.

The phylogenetic tree of the Nora-like viruses tends to mirror that of their hosts (Fig. 7), suggesting that this virus family is old and that the viruses tend to keep narrow host ranges. Occasional shifts in host range must have happened, the most dramatic one involving a sea anemone. It should be stressed, though, that most of these sequences come from large metagenomic projects, and the exact links between the viruses and the organisms in which they were found are still uncertain. For instance, Fopius arisanus is a parasitoid wasp, feeding on Bactrocera dorsalis and Ceratitis capitata, and the virus may well derive from the gut contents of this wasp. Similarly, dragon flies (Odonata) are voracious predators on flies and other flying insects, and their viromes may include viruses present in their diet. A similar argument could be made about the spider. It is of course also possible that the viruses have adapted to replicate in these predators. However, the sea anemone remains a mystery.

In conclusion, we showed that the Nora virus has a $\mathrm{T}=1$ arrangement where VP4C is present around the five-fold axes of symmetry, VP4A around the two-fold axes of symmetry and VP4B around the three-fold axes of symmetry. Each protein shows the $\beta$-jelly roll fold characteristic of Picornavirales, but with an $\alpha$-helical domain protrusion from the virion surface and an unusual interaction of two N-termini from symmetry-related VP4A around the two-fold axes. Taken together, both global and detailed analysis of the capsid structure, the genome organization and the genetic distance to other viruses, suggest that Nora virus and the clade of related viruses can be described as representatives of a new virus family within the order Picornavirales.

\section{Materials and methods}

Production and purification of Nora virus. Persistent viral infections are common in Drosophila ${ }^{24}$. To avoid cross-contamination with other viruses, dechorionated Drosophila melanogaster eggs were infected with Nora virus as described earlier ${ }^{9}$. Nora virus was propagated and purified essentially as described earlier ${ }^{1-3,9}$.

CryoEM and image processing. Aliquots $(3 \mu \mathrm{l})$ of purified virus in $10 \mathrm{mM}$ Tris- $\mathrm{HCl} \mathrm{pH}$ 7.4. buffer were vitrified in a Leica EM GP device at $22{ }^{\circ} \mathrm{C}$ and $70 \%$ humidity on glow discharged Quantifoil $2 / 2$ holey carbon grid in liquid ethane. CryoEM data were collection at eBIC at the Diamond Light Source, UK on a FEI Krios 

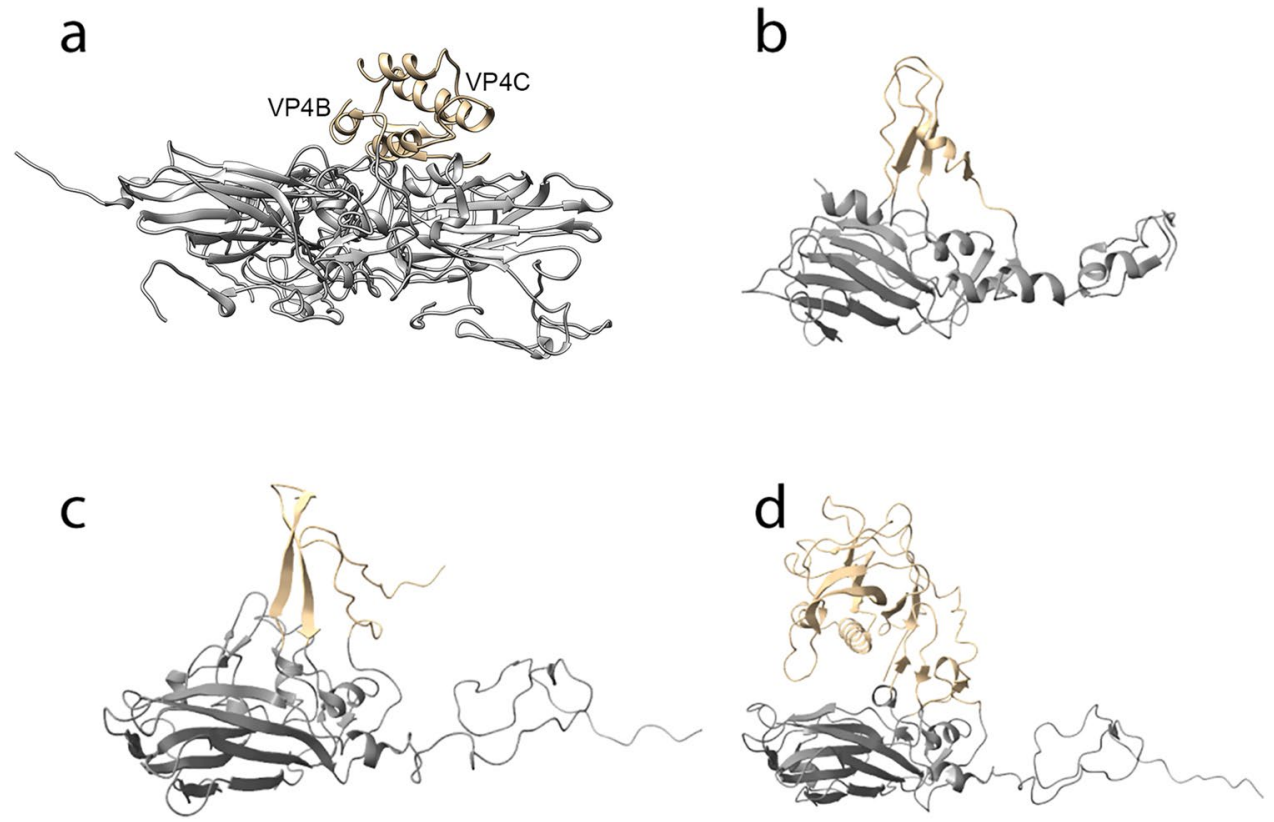

Figure 4. Surface protrusion comparison. The surface protrusions (gold) on the (a) Nora virus, (b) triatoma virus, (c) Israel acute paralysis virus and (d) slow bee paralysis virus are significantly different from each other. The Nora virus VP4C and VP4B C-termini form an $\alpha$-helix-bundle. Triatoma has the least pronounced protrusion, made by VP1, a capsid protein present around the vertices. It is composed of $\beta$-hairpin and a short helix. Israel acute paralysis virus has long $\beta$-strands protruding out from the capsid protein VP2 found around the three-fold axes. The capsid protein VP3 C-terminus in slow bee paralysis virus forms a surface protrusion made of $\beta$-sheets and two helices. The VP3 is protein present around the three-fold symmetry axes. Only the position in Nora virus and triatoma virus is similar.
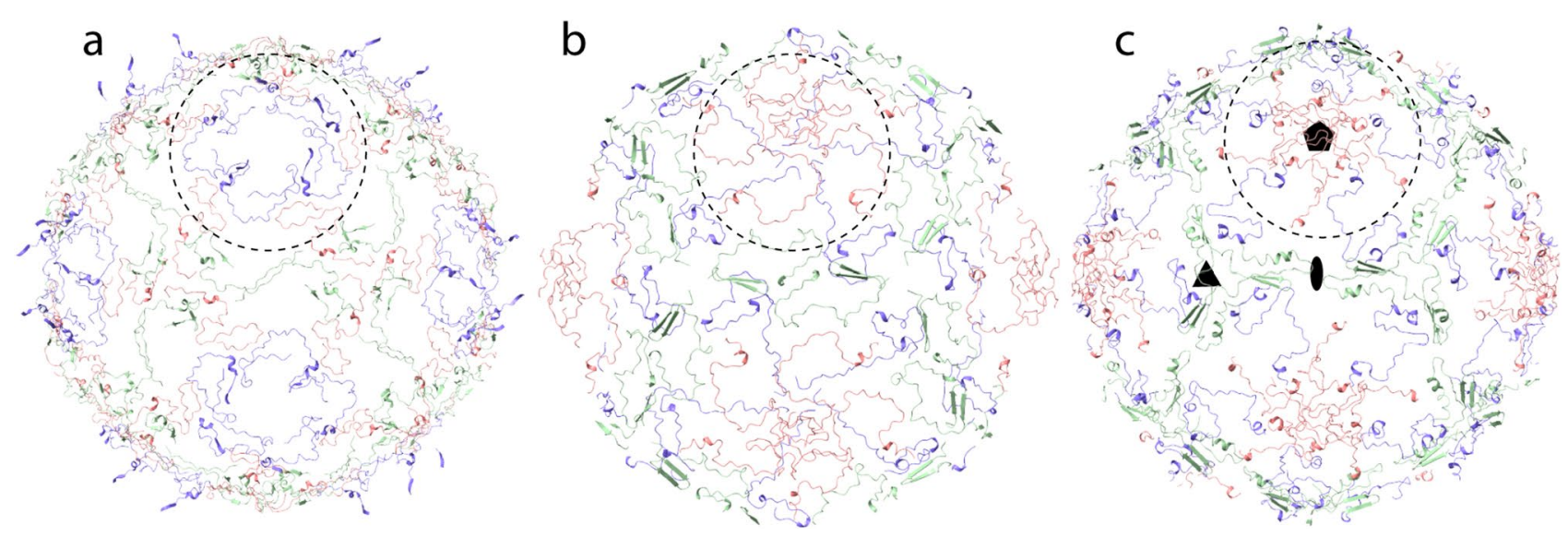

Figure 5. Network of capsid protein $\mathrm{N}$-termini. The first $50 \mathrm{~N}$-terminus residues present in the atomic models of Nora virus (a), CVA9 PDB ID: 1d4m (b) and HPeV1 PDB ID 4z92 (c) are shown. Green ribbon is VP4A in Nora virus, VP1 in CVA9 and VP0 in HPeV1. Pink ribbon: is VP4B in Nora virus and VP3 in CVA9 and $\mathrm{HPeV} 1$. Blue ribbon is VP4C in Nora virus and VP1 in CVA9 and HPeV1. Only the front half of the capsids are shown for clarity. Black ellipse, pentagon and triangles indicate positions of two-fold, five-fold and three-fold symmetry axes in (a)-(c). The dotted circle indicates the region which stabilizes the pentamer within the capsid.

$300 \mathrm{kV}$ TEM equipped with a Gatan post-GIF K2 Summit detector. The GIF was set to $20 \mathrm{eV}$ slit width and FEI EPU software was used to automatically the data. Each exposure was written out as a 20 frames stack with an estimated total electron dose of $40 \mathrm{e}^{-} / \AA^{2}$ and a sampling of $1.06 \AA$ /pixel. The initial dataset consisted of 3516 frame stacks, and the frames were aligned prior to processing using motioncorr ${ }^{25}$. We used Ethan ${ }^{26}$ for automated particle picking, CTFFIND4 for CTF estimation and correction ${ }^{27}$. An initial model was built with 150 picked particles using random model generation module in $\mathrm{AUTO} \mathrm{DEM}^{28}$ with icosahedral-symmetry imposed. Both $2 \mathrm{D}$ and $3 \mathrm{D}$ classification in Relion version $1.3^{29}$, were used to reduce the dataset heterogeneity. A total of 16,131 particles were refined using the $3 \mathrm{D}$ autorefine module, followed by particle polishing, an additional $3 \mathrm{D}$ autore- 
a
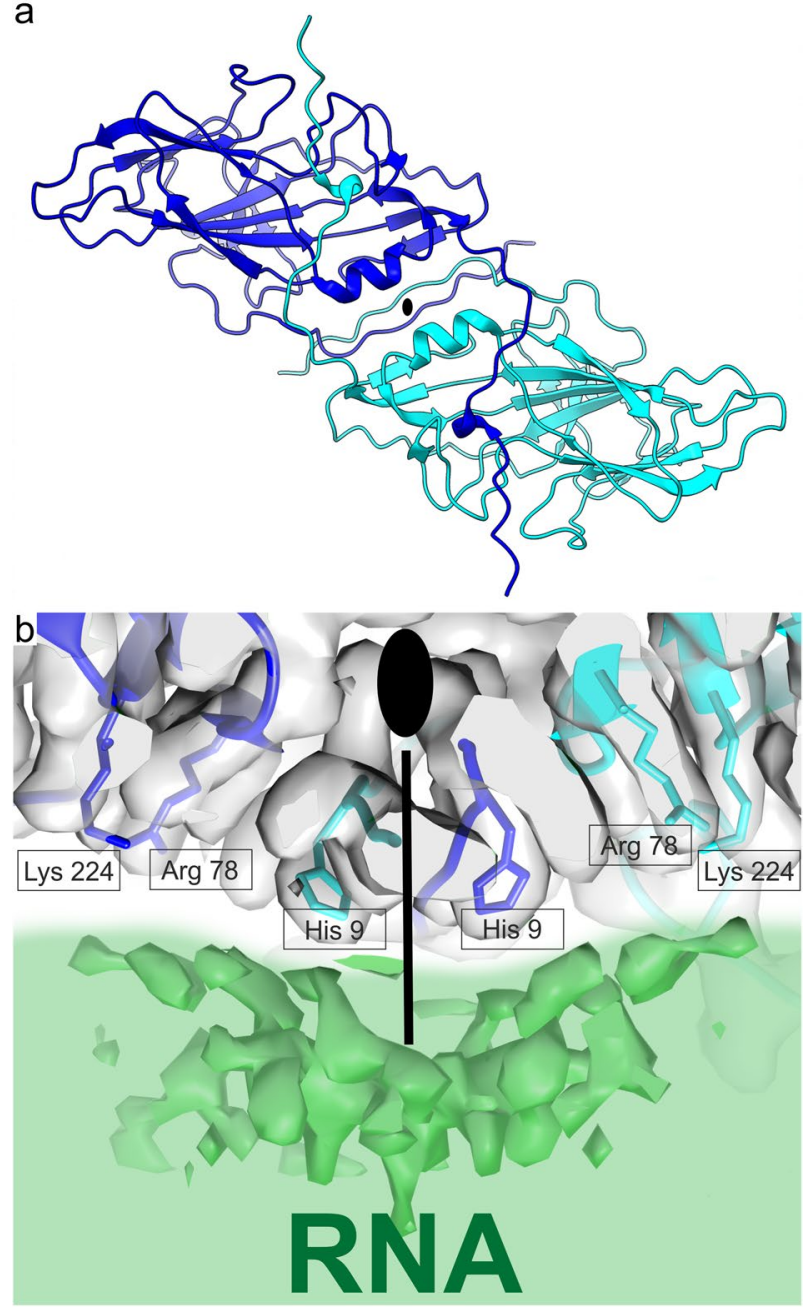

Figure 6. Two-fold symmetry axis. (a) VP4A dimer shown from outside the capsid. (b) VP4A interaction with RNA; a close-up view of the area depicted by white arrow in Fig. 1b showing interaction between VP4A and RNA genome below the twofold symmetry axis. Grey semi-transparent surface: reconstructed VP4A volume rendered at 2.5 SD above mean. Green surface: RNA-density rendered at 2.5 SD above mean. The green wash represents the interior of the capsid. (a,b) Cyan and blue ribbon: atomic models of the two twofold associated VP4A-chains, positively charged VP4A side chains are shown and labelled. Black ellipse and line indicate position of the twofold symmetry axis.

fine step, and finally B-factor correction in the "post processing" module using a B-factor value of - 20. The final refinement step combining two independent datasets gave a resolution of $2.7 \AA$ as assessed by the 0.143 criterion Fourier shell correlation from the deposited half maps using the EMDB server (EMDB-3528; Fig. S2).

Model building and refinement. We used the "Volume tracer"-tool in UCSF Chimera ${ }^{30}$ to trace C- $\alpha$ backbones of the constituent proteins from capsid density. Volumes corresponding to the three distinct subunit densities were then segmented with "Zone"-tool in UCSF Chimera at a radius of $8 \AA$. The models were built de novo into each segmented EM density in $\mathrm{COOT}^{31}$ using the known amino acid sequences of VP4A, VP4B and VP4C. The models were refined in real space using Phenix ${ }^{32}$ and in Fourier space using Refmac ${ }^{33}$. This step was iterated with local refinements in COOT until no further improvement in the refinement statistics were observed (Table 1). We combined the atomic models of the three subunits into the full asymmetric unit, which was consequently re-refined in Phenix to resolve any clashes within the asymmetric unit. The UCSF Chimera sym command was used to build the whole virus capsid. UCSF Chimera or ChimeraX ${ }^{34}$ were used for all visualizations. 


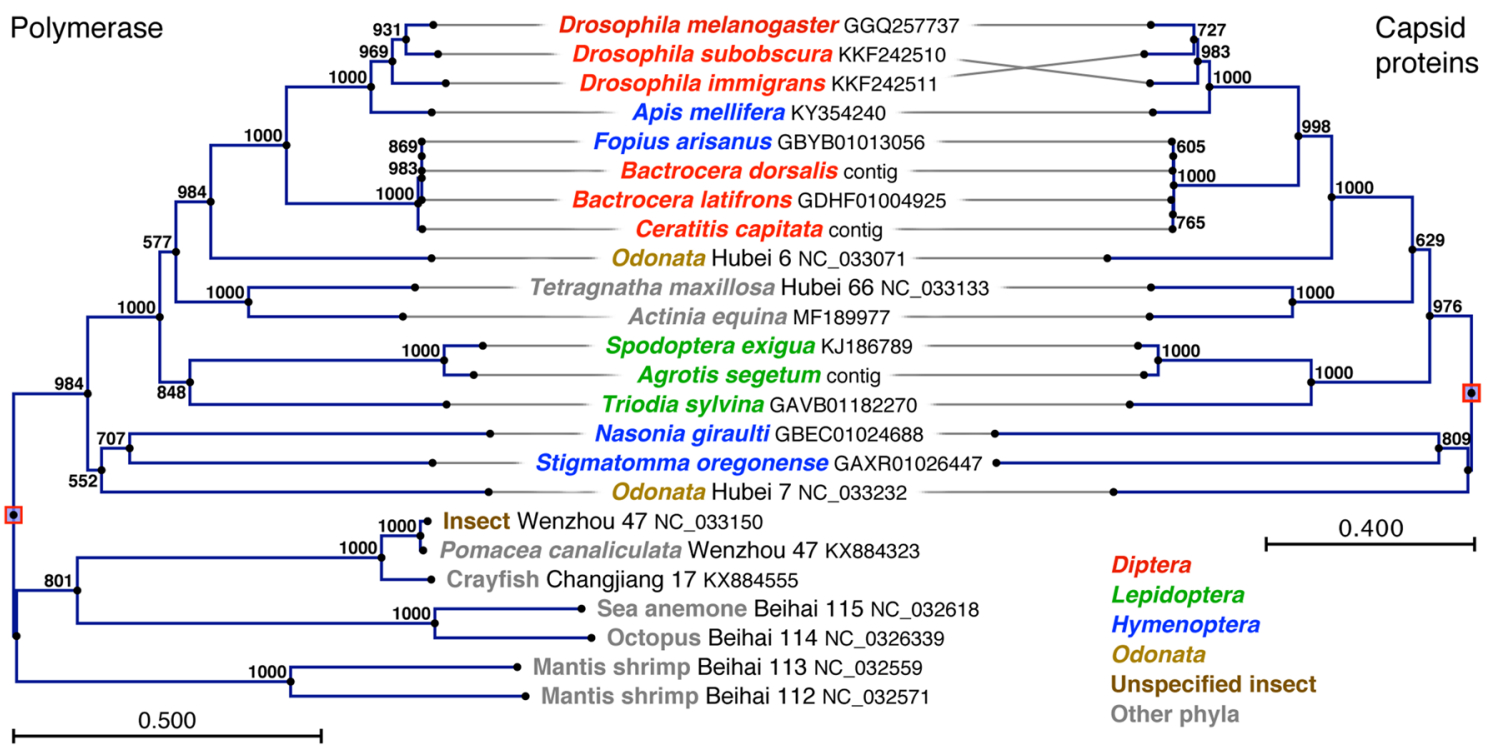

Figure 7. Phylogenetic analysis of Nora-like viruses. Nora VP4-like sequences were retrieved from the NCBI $\mathrm{nr} / \mathrm{nt}$, EST and TSA databases by pblast and tblastn searches. If possible, contigs were constructed from overlapping short TSA and EST sequences. Single short partial sequences were excluded from further analysis. Phylogenetic trees were reconstructed from conserved regions in the polymerase (left) and VP4 (right) amino acid sequences, using the neighbor-joining algorithm of the CLC Main Workbench package, version 6.7.1. As an outgroup for the polymerases we used members of the Nora Virus Related Clade, described by Shi et al. ${ }^{8}$, that lack Nora VP4-like capsid proteins.

Structural alignments and buried surface area calculation. The structural alignments were performed on the DALI web server ${ }^{13}$. The buried surface area for all the viruses were obtained from VIPERdb association energy data analysis ${ }^{35}$.

\section{Data availability}

The datasets generated during the current study are freely available in the following public repositories: the density map of the Nora virus reconstruction has been deposited in the Electron Microscopy Data Bank under the accession codes EMD-3528 https:/www.ebi.ac.uk/pdbe/entry/emdb/EMD-3528. The atomic models for Nora virus have been deposited in the Protein Databank in Europe with the PDB ID: $5 \mathrm{~mm} 2 \mathrm{https}: / /$ www.ebi. ac.uk/pdbe/entry/pdb/5mm2. All the raw data collected for the Nora virus reconstruction are available through the Electron Microscopy Pilot Image Archive under the accession code EMPIAR-10088 https://www.ebi.ac.uk/ pdbe/emdb/empiar/.

Received: 3 July 2020; Accepted: 26 October 2020

Published online: 12 November 2020

\section{References}

1. Habayeb, M. S., Ekengren, S. K. \& Hultmark, D. Nora virus, a persistent virus in Drosophila, defines a new picorna-like virus family. J. Gen. Virol. 87, 3045-3051 (2006).

2. Habayeb, M. S. et al. The Drosophila Nora virus is an enteric virus, transmitted via feces. J. Invertebr. Pathol. 101, 29-33 (2009).

3. Habayeb, M. S., Ekström, J. O. \& Hultmark, D. Nora virus persistent infections are not affected by the RNAi machinery. PLoS ONE 4, e5731 (2009).

4. Cordes, E. J., Licking-Murray, K. D. \& Carlson, K. A. Differential gene expression related to Nora virus infection of Drosophila melanogaster. Virus Res. 175, 95-100 (2013).

5. Ekström, J. O. \& Hultmark, D. A novel strategy for live detection of viral infection in Drosophila melanogaster. Sci. Rep. 6, 26250. https://doi.org/10.1038/srep26250 (2016)

6. Le Gall, O. et al. Picornavirales, a proposed order of positive-sense single-stranded RNA viruses with a pseudo-T $=3$ virion architecture. Arch. Virol. 153, 715-727 (2008).

7. Oliveira, D. C. S. G. et al. Data mining cDNAs reveals three new single stranded RNA viruses in Nasonia (Hymenoptera: Pteromalidae). Insect. Mol. Biol. 19(Suppl 1), 99-107. https://doi.org/10.1111/j.1365-2583.2009.00934.x (2010).

8. Shi, M. et al. Redefining the invertebrate RNA virosphere. Nature 540, 539-543 (2016).

9. Ekström, J. O. et al. Drosophila Nora virus capsid proteins differ from those of other picorna-like viruses. Virus Res. 160, 51-58 (2011).

10. Remnant, E. J. et al. A diverse range of novel RNA viruses in geographically distinct honey bee populations. J. Virol. 91, e00158-e217. https://doi.org/10.1128/JVI.00158-17 (2017).

11. Medd, N. C. et al. The virome of Drosophila suzukii, an invasive pest of soft fruit. Virus Evol. 4, vey009. https://doi.org/10.1093/ ve/vey009 (2018).

12. Sadanandan, S. A., Ekström, J. O., Jonna, V. R., Hofer, A. \& Hultmark, D. VP3 is crucial for the stability of Nora virus virions. Virus Res. 223, 20-27 (2016).

13. Holm, L. \& Laakso, L. M. Dali server update. Nucleic Acids Res. 44, W351-W355. https://doi.org/10.1093/nar/gkw357 (2016). 
14. Hesketh, E. L. et al. Mechanisms of assembly and genome packaging in an RNA virus revealed by high-resolution cryo-EM. Nat. Commun. 6, 10113. https://doi.org/10.1038/ncomms10113 (2015).

15. Wang, X. et al. A sensor-adaptor mechanism for enterovirus uncoating from structures of EV71. Nat. Struct. Mol. Biol. 19, 424-429 (2012).

16. Shakeel, S. et al. Structural and functional analysis of coxsackievirus A9 integrin alphavbeta6 binding and uncoating. J. Virol. 87, 3943-3951 (2013).

17. Seitsonen, J. J. et al. Structural analysis of coxsackievirus A7 reveals conformational changes associated with uncoating. J. Virol. 86, 7207-7215 (2012)

18. Kotecha, A. et al. Structure-based energetics of protein interfaces guides foot-and-mouth disease virus vaccine design. Nat. Struct. Mol. Biol. 22, 788-794 (2015).

19. Shakeel, S. et al. Genomic RNA folding mediates assembly of human parechovirus. Nat. Commun. 8, 5. https://doi.org/10.1038/ s41467-016-0011-z (2017).

20. Shakeel, S. et al. Multiple capsid-stabilizing interactions revealed in a high-resolution structure of an emerging picornavirus causing neonatal sepsis. Nat. Commun. 7, 11387. https://doi.org/10.1038/ncomms11387 (2016).

21. Hesketh, E. L., Meshcheriakova, Y., Thompson, R. F., Lomonossoff, G. P. \& Ranson, N. A. The structures of a naturally empty cowpea mosaic virus particle and its genome-containing counterpart by cryo-electron microscopy. Sci. Rep. 7, 539. https://doi. org/10.1038/s41598-017-00533-w (2017).

22. Pickl-Herk, A. et al. Uncoating of common cold virus is preceded by RNA switching as determined by X-ray and cryo-EM analyses of the subviral A-particle. Proc. Natl. Acad. Sci. U.S.A. 110, 20063-20068 (2013).

23. Dai, X. et al. In situ structures of the genome and genome-delivery apparatus in a single-stranded RNA virus. Nature 541, 112-116 (2017).

24. Ambrose, R. L. et al. Drosophila A virus is an unusual RNA virus with a $\mathrm{T}=3$ icosahedral core and permuted RNA-dependent RNA polymerase. J. Gen. Virol. 90, 2191-2200 (2009).

25. Li, X. et al. Electron counting and beam-induced motion correction enable near-atomic-resolution single-particle cryo-EM. Nat. Methods 10, 584-590 (2013).

26. Kivioja, T., Ravantti, J., Verkhovsky, A., Ukkonen, E. \& Bamford, D. Local average intensity-based method for identifying spherical particles in electron micrographs. J. Struct. Biol. 131, 126-134 (2000).

27. Rohou, A. \& Grigorieff, N. CTFFIND4: fast and accurate defocus estimation from electron micrographs. J. Struct. Biol. 192, 216-221 (2015).

28. Yan, X., Sinkovits, R. S. \& Baker, T. S. AUTO3DEM-an automated and high throughput program for image reconstruction of icosahedral particles. J. Struct. Biol. 157, 73-82 (2007).

29. Scheres, S. H. W. RELION: implementation of a Bayesian approach to cryo-EM structure determination. J. Struct. Biol. 180, 519-530 (2012).

30. Pettersen, E. F. et al. UCSF Chimera-a visualization system for exploratory research and analysis. J. Comput. Chem. 25, 1605-1612 (2004).

31. Emsley, P., Lohkamp, B., Scott, W. G. \& Cowtan, K. Features and development of Coot. Acta Crystallogr. D Biol. Crystallogr. 66, 486-501 (2010).

32. Adams, P. D. et al. PHENIX: a comprehensive Python-based system for macromolecular structure solution. Acta Crystallogr. D Biol. Crystallogr. 66, 213-221 (2010).

33. Murshudov, G. N., Vagin, A. A. \& Dodson, E. J. Refinement of macromolecular structures by the maximum-likelihood method. Acta Crystallogr. D Biol. Crystallogr. 53, 240-255 (1997).

34. Goddard, T. D. et al. UCSF ChimeraX: meeting modern challenges in visualization and analysis. Protein Sci. 27, $14-25$ (2018).

35. Carrillo-Tripp, M. et al. VIPERdb2: an enhanced and web API enabled relational database for structural virology. Nucleic Acids Res. 37, D436-442 (2009).

36. Kucukelbir, A., Sigworth, F. J. \& Tagare, H. D. Quantifying the local resolution of cryo-EM density maps. Nat. Methods 11, 63-65 (2014).

\section{Acknowledgements}

We would like to thank Daniel Clare for support at the Diamond Light Source and Eevakaisa Vesanen for excellent technical assistance. We acknowledge the Diamond Light Source for access to the cryoEM facilities at the UK national electron bio-imaging centre (eBIC), proposal EM14263-1, funded by the Wellcome Trust, MRC and BBSRC. The CSC-IT Center for Science Ltd., Biocenter Finland, Instruct-ERIC Centre Finland and Instruct-FI cryoEM unit provided facilities. This study was supported by the Academy of Finland (275199, 315950, 328112 to SJB, 252384 to DH), the Sigrid Juselius Foundation (SJB, DH) and the Swedish Research Council (DH).

\section{Author contributions}

J.E., D.H. and S.J.B. conceived the idea. P.L., S.S., J.E. and S.J.B. designed the experiments. P.L., S.S., J.E. and P.M. carried out the experiments. All authors contributed to interpretation of results and writing of the manuscript.

\section{Competing interests}

The authors declare no competing interests.

\section{Additional information}

Supplementary information is available for this paper at https://doi.org/10.1038/s41598-020-76613-1.

Correspondence and requests for materials should be addressed to S.J.B.

Reprints and permissions information is available at www.nature.com/reprints.

Publisher's note Springer Nature remains neutral with regard to jurisdictional claims in published maps and institutional affiliations. 
(c) (i) Open Access This article is licensed under a Creative Commons Attribution 4.0 International cc) License, which permits use, sharing, adaptation, distribution and reproduction in any medium or format, as long as you give appropriate credit to the original author(s) and the source, provide a link to the Creative Commons licence, and indicate if changes were made. The images or other third party material in this article are included in the article's Creative Commons licence, unless indicated otherwise in a credit line to the material. If material is not included in the article's Creative Commons licence and your intended use is not permitted by statutory regulation or exceeds the permitted use, you will need to obtain permission directly from the copyright holder. To view a copy of this licence, visit http://creativecommons.org/licenses/by/4.0/.

(C) The Author(s) 2020 